\title{
Modal dynamics above the threshold of higher-order transverse modes in a vertical-cavity surface-emitting laser with isotropic optical feedback
}

\author{
Hong Lin, ${ }^{1, *}$ Erik G. Born, ${ }^{1}$ Nola J. Palombo, ${ }^{1}$ and Angel Valle ${ }^{2}$ \\ ${ }^{1}$ Department of Physics and Astronomy, Bates College, Lewiston, Maine 04240, USA \\ ${ }^{2}$ Instituto de Fisica de Cantabria (CSIS-UC), Edificio Juan Jorda, Campus Universitario, Avada Los Castros s/n, \\ E-39005 Santander, Spain \\ *Corresponding author: hlin@bates.edu
}

Received April 26, 2010; revised August 20, 2010; accepted August 21, 2010; posted August 26, 2010 (Doc. ID 127558); published October 18, 2010

We have experimentally studied modal dynamics of a vertical-cavity surface-emitting laser (VCSEL) above the threshold of higher-order transverse modes when the VCSEL is subject to isotropic optical feedback. The dynamics of the higher-order mode reveals resemblance to the so-called low-frequency fluctuation (LFF) and the transition from LFF to coherence collapse near the threshold of single-transverse-mode VCSEL. Influence of control parameters on the dynamics is investigated, and possible mechanism of the dynamics is discussed. (C) 2010 Optical Society of America

OCIS codes: $140.7260,190.3100$.

\section{INTRODUCTION}

The vertical-cavity surface-emitting laser (VCSEL) has proven to be a useful device in optical data communications and other applications because of its singlelongitudinal-mode operation, circular beam profile, low threshold current, and wide bandwidth of modulation. Similar to edge-emitting lasers, however, the VCSEL is sensitive to optical feedback that arises inevitably from any surface in the path of the output beam [1,2]. Optical feedback can induce a variety of intensity and polarization instabilities, for instance, low-frequency fluctuation (LFF), coherence collapse (CC), polarization selfmodulation, and polarization switching [3-6]. Indeed, VCSELs with optical feedback have attracted strong research interests from perspectives of both fundamental sciences and applications.

Because of the large diameter of its cavity, a VCSEL is not always in single-transverse-mode operation. Usually, the VCSEL operates in a fundamental mode near its threshold. The fundamental mode may have two orthogonally polarized components with a $10-20 \mathrm{GHz}$ frequency difference. The VCSEL often switches from one polarization to the other in the single-transverse-mode regime with varying injection current. As the injection current increases, higher-order transverse modes can emerge; their polarizations can be parallel or perpendicular to that of the fundamental mode. A lot of experimental and theoretical studies have been conducted on the dynamics of single-transverse-mode VCSELs subject to optical feedback [4-13]. In-phase and antiphase fluctuations are manifested in orthogonal polarizations. Affected by dichroism, LFF is observed in both single polarization and orthogonal polarizations near the threshold [9]. The de- pendence of LFF on control parameters (e.g., injection current, optical feedback level, and length of external cavity) has been measured and compared to the theory $[9,12]$. With the increasing current, the LFF dropouts become more frequent and eventually indistinguishable, indicating that the laser reaches CC $[4,9,10]$. This is the same dynamical route as edge-emitting lasers $[14,15]$.

Meanwhile, investigation on dynamics of multitransverse-mode VCSELs involves more complexity [16-20]. The numerical results show that VCSELs enter CC when feedback is strong enough [16]; for weak feedback, transverse dynamics can change from antiphase to in-phase as the injection current is varied [17]. While orthogonal states of the total output are anticorrelated, spatially resolved measurements reveal that correlation property between orthogonal components might be different for different transverse modes [18], which is in agreement with the numerical results obtained in [19]. It has also been shown experimentally and theoretically that frequency-selective polarized feedback can increase the intensity of the selected mode and suppress instability in the emission [20]. However, dynamics of each transverse mode, both intensity and polarization, has not been fully investigated experimentally. Although efforts were made to determine modal dynamics in [18], the aperture used for spatially resolved measurement was unable to isolate one transverse mode from the others. In order to study the dynamics of each transverse mode, it is necessary to use a more effective method for mode selection.

In this paper we report an experimental investigation on dynamics of individual transverse modes near the threshold of higher-order transverse modes when the VCSEL is subject to isotropic optical feedback. Despite 
the same type of feedback as in [18], now the substrate temperature is introduced as a parameter. The modes are frequency selected by using an etalon, which allows us to study dynamics of each transverse mode. Our investigation reveals that for a certain range of temperature, the dynamics of the first-order mode is similar to the so-called LFF and LFF-CC near the threshold of semiconductor lasers [15]. In particular, LFF features are manifested in the higher-order mode when the injection current is $\sim 2.0$ to 2.3 times the threshold current. To our knowledge, this is the first observation that higher-order transverse modes can demonstrate LFF dynamics. Influences of injection current, strength of feedback, and substrate temperature on this dynamical regime are explored. In Section 2 we describe the experimental setup and the efficiency of mode selection. Our results are reported in Section 3. Section 4 gives the discussion and conclusion.

\section{EXPERIMENTAL SETUP AND MODE SELECTION}

A proton-implanted VCSEL (Honeywell HFE-4083, 844 $\mathrm{nm}$ ) is used in our experiments. The temperature of the VCSEL is stabilized within $0.01^{\circ} \mathrm{C}$ using a temperature controller (Thorlabs TEC2000). The experimental setup is shown in Fig. 1, in which the output of the VCSEL is collimated with a lens (CL) of $f=8 \mathrm{~mm}$. With a nonpolarizing beam splitter, $\mathrm{BS}_{1}, 50 \%$ of the output is sent to a total reflector, M, which provides optical feedback to the VCSEL. The other $50 \%$ of the output is used for observation and measurements. The external cavity length is $31 \mathrm{~cm}$. The strength of optical feedback is changed by inserting a neutral density filter $\left(\mathrm{ND}_{3}\right)$ in the external cavity. The strongest feedback is achieved when $\mathrm{ND}_{3}$ is not inserted. We refer to this feedback as isotropic feedback because there are no elements in the external cavity for polarization selection, frequency selection, or spatial filtering. Since the VCSEL operates with several transverse modes, however, the strength of feedback may be different for different modes. The optical spectrum and beam pattern of the VCSEL are separately monitored with a Fabry-Perot (F-P) spectrum analyzer $(\mathrm{FSR}=750 \mathrm{GHz})$ and a chargecoupled-device (CCD) camera. A neutral density filter $\left(\mathrm{ND}_{1}\right)$ is placed in front of the $\mathrm{F}-\mathrm{P}$ spectrum analyzer to minimize any unwanted feedback. Another neutral den-

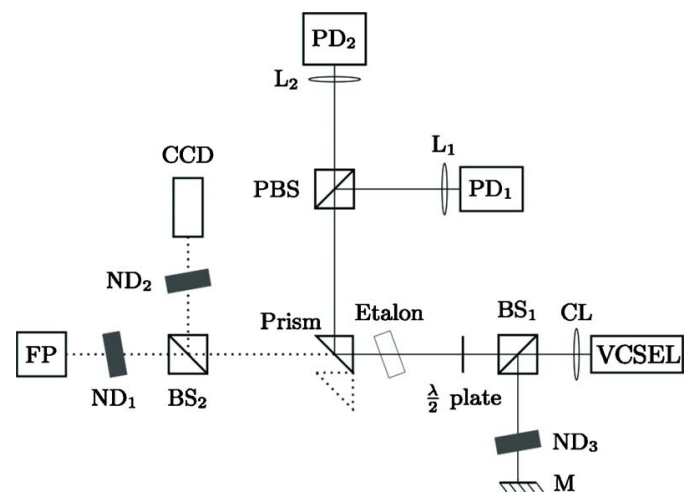

Fig. 1. Experimental setup, where BS stands for nonpolarizing beam splitter, $\mathrm{M}$ for feedback mirror, PBS for polarizing beam splitter, PD for photodetector, and ND for neutral density filter. sity filter $\left(\mathrm{ND}_{2}\right)$ is used with the CCD camera to avoid intensity saturation. For polarization resolving measurements, a half-wave plate and a polarizing beam splitter (PBS) are used to separate two orthogonal polarizations. Photodetectors $\mathrm{PD}_{1}$ and $\mathrm{PD}_{2}$ (New Focus 1601) measure $y$ - and $x$-polarized states, respectively, where $y$ polarization refers to the polarization perpendicular to the optical table, and $x$ polarization is parallel to the optical table. The bandwidth of the photodetectors is $1 \mathrm{GHz}$. The alternating current (ac) outputs of the detectors are sent to a digital oscilloscope (Tektronix DPO 7254, bandwidth of $2.5 \mathrm{GHz}$ ) to observe temporal fluctuations; the direct current (dc) outputs reflect the average power of the polarized states. The typical sampling rate is $20 \mathrm{GS} / \mathrm{s}$. The power spectrum of each polarized state is monitored by sending the ac output of the corresponding detector to a rf spectrum analyzer (Tektronix 2712, bandwidth of 1.8 $\mathrm{GHz}$ ). When observing the nonpolarizing signal, the PBS is removed, and $\mathrm{PD}_{2}$ is used for measurements. An etalon is used for mode selection. The free spectral range of the etalon is $380 \mathrm{GHz}$. The power of each mode after being selected by the etalon has a significant loss as shown in Fig. 2. To make sure that the light is strong enough to be detected after the etalon, we use a prism, instead of a nonpolarizing beam splitter, installed on a translational stage controlled with a micrometer. For each transverse mode, we monitor its optical spectrum to get the optimal selection. Then the prism is moved in to send the light to the detectors.

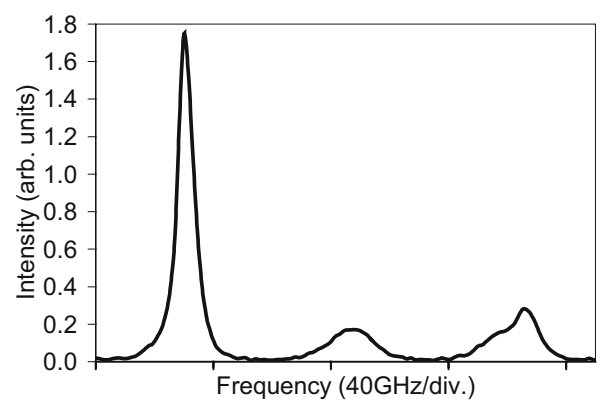

(a)

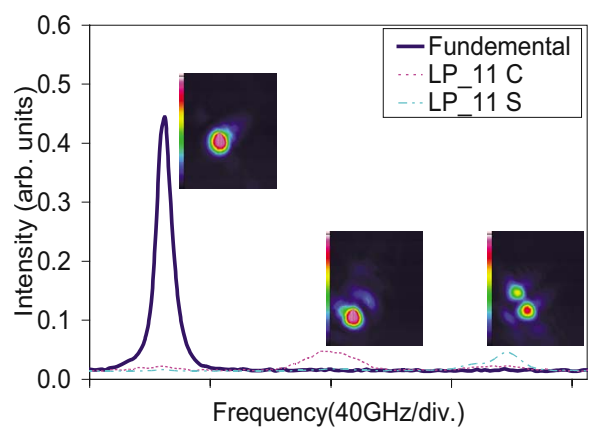

(b)

Fig. 2. (Color online) Mode selection using an etalon. (a) Optical spectrum of the VCSEL with the strongest feedback before mode selection. (b) Optical spectra of the selected modes, in which the solid, dark blue curve is for $\mathrm{LP}_{01}$ mode, the dotted pink curve is for $\mathrm{LP}_{11}^{c}$ mode, and the dashed, light blue curve is for $\mathrm{LP}_{11}^{s}$ mode. The insets are spatial profiles of the corresponding frequency peak. The spatial profiles of the $\mathrm{LP}_{01}$ and $\mathrm{LP}_{11}^{s}$ modes were attenuated to avoid saturation at the CCD camera. The substrate temperature is $64^{\circ} \mathrm{C}$. 
Several substrate temperatures, from $T=24.0^{\circ} \mathrm{C}$ to $T$ $=64.0^{\circ} \mathrm{C}$, have been used in our experiments. For each chosen temperature, the VCSEL is set at the temperature for at least $15 \mathrm{~h}$ before making measurements. As is known, substrate temperature affects threshold current, directions of polarizations, and polarization switching [21-23]. This is because increasing temperature causes both the cavity resonance and laser gain profile to shift to longer wavelengths due to the refractive index and bandgap temperature dependence, but the laser gain shifts faster than the cavity resonance [21]. Depending on the spectral shift between the cavity resonance and laser gain, the threshold may decrease or increase. The threshold of our VCSEL increases with increasing temperature. This implies that the operating wavelength is moving away from the peak of the laser gain. The directions of polarizations remain unchanged in the temperature range we have used. When the injection current $I$ is increased, higher-order transverse modes start lasing. The thresholds of higher-order transverse modes also increase with the temperature. Except for the increase in thresholds, most dynamical features that we are reporting are similar for all the temperatures. Unless otherwise specified, the presented results in this paper were obtained at $34^{\circ} \mathrm{C}$.

For $T=34^{\circ} \mathrm{C}$, the threshold current of the solitary VC$\mathrm{SEL}, I_{\text {th } 0}$, is $2.87 \mathrm{~mA}$. The VCSEL is $y$-polarized until polarization switching, when the VCSEL's polarization changes from $y$ - to $x$-component at $\sim 3.28 \mathrm{~mA}$, and remains $x$-polarized until the onset of higher-order transverse modes. The frequency difference between these two orthogonally polarized components is about $10 \mathrm{GHz}$, where the $y$-component has a higher frequency. The VCSEL operates in a single-transverse-mode for $I<1.5 I_{\text {th } 0}$. The beam pattern reveals that the mode is a fundamental mode, or $\mathrm{LP}_{01}$ mode. For $I \geq 4.29 \mathrm{~mA}\left(I / I_{\mathrm{th} 0} \geq 1.5\right)$, a higher-order transverse mode begins lasing. This mode is essentially $x$-polarized near its threshold. The frequency of this transverse mode, $\nu_{1}$, is $\sim 110 \mathrm{GHz}$ higher than the frequency of the fundamental mode, $\nu_{0}$. When $I$ $=4.89 \mathrm{~mA}\left(I / I_{\mathrm{th} 0}=1.7\right)$, another transverse mode of frequency $\nu_{2}$ is on, and $\nu_{2}-\nu_{0}$ is $\sim 65 \mathrm{GHz}$. At the same time, the power of the $y$ polarization of the total output starts to increase. This indicates that the transverse mode of frequency $\nu_{2}$ is $y$-polarized near its threshold, which is confirmed by the polarization resolved optical spectrum. For higher currents, the fundamental mode is still essentially $x$-polarized, but the two higher-order transverse modes may have two orthogonally polarized components with comparable powers. When $I / I_{\text {th } 0}$ is $\sim 2.9$, the fourth transverse mode is on. In our study, we focused on the regime in which the VCSEL operates with two or three different transverse modes.

When the strongest optical feedback is applied, the threshold is reduced by $\sim 0.11 \mathrm{~mA}$ or $4 \%$. Reductions in the threshold of $\nu_{1}$ and $\nu_{2}$ are $\sim 0.01$ and $0.26 \mathrm{~mA}$, respectively. The difference between the threshold reductions may be related to different spatial profiles of the two modes shown in Fig. 2(b). Comparing to the solitary VCSEL, the power of the $x$-state of the total output is decreased, whereas the power of the $y$-state is increased, implying that optical feedback stimulates $y$ polarization. This is probably because the external cavity is aligned at the threshold where the VCSEL is $y$-polarized. However, the $x$-state is still the dominant polarization.

Figure 2 gives an example of mode selection in the three-mode regime. Figure 2(a) is the optical spectrum of the VCSEL with the strongest feedback for $7.5 \mathrm{~mA}$ at $64^{\circ} \mathrm{C}$, and Fig. 2(b) illustrates the three selected modes under the same conditions, where the insets are beam patterns of the corresponding modes. The strongest peak in the optical spectrum is the $\mathrm{LP}_{01}$ mode. The mode of frequency $\nu_{1}$ can be described as a $\mathrm{LP}_{11}^{s}$ mode, and the mode of $\nu_{2}$ is a $\mathrm{LP}_{11}^{c}$ mode [24]. However, the intensity of one lobe of the $\mathrm{LP}_{11}^{c}$ mode is much stronger than the other, which may result from some internal asymmetry of the VCSEL. The spatial profiles of the $\mathrm{LP}_{01}$ and $\mathrm{LP}_{11}^{s}$ modes have a stronger overlap, whereas the dominant lobe of the $\mathrm{LP}_{11}^{c}$ mode is far from the spatial profiles of the other two transverse modes. We think that the different threshold reductions for the two first-order modes may result from different spatial profiles of the two modes that result in different spatial overlaps with the feedback beam. Because of the power loss caused by inserting the etalon, it is difficult to measure the dynamics of the $\mathrm{LP}_{11}^{c}$ mode and the weaker polarization of the $\mathrm{LP}_{01}$ and $\mathrm{LP}_{11}^{s}$ modes for most cases.

\section{DYNAMICS NEAR THE THRESHOLD OF THE HIGHER-ORDER TRANSVERSE MODES}

With the strongest feedback, the VCSEL demonstrates typical LFF near the threshold. When the current is above $3.0 \mathrm{~mA}\left(I / I_{\mathrm{th} 0}>1.04\right)$, the fluctuations become more and more irregular with increasing current. For $I$ $\geq 3.10 \mathrm{~mA}$, the features of LFF completely vanish. This is a well known dynamical route for single-transverse-mode VCSEL and other edge emitters with optical feedback: the lasers evolve from LFF to CC regime with increasing injection currents $[4,9,10,14,15]$. The fluctuations of $x$ - and $y$-components of the $\mathrm{LP}_{01}$ mode are anticorrelated. When the current ranges from 3.5 to $4.1 \mathrm{~mA}$, however, the feedback-induced fluctuations become minimal; only a weak narrow peak corresponding to the external cavity resonance appears in the power spectrum of the output. This may imply that the frequency of the VCSEL in this current range does not form effective resonance in the external cavity [7]. The external cavity resonance peak in the power spectrum is widened and begins to increase when $I>4.1 \mathrm{~mA}$.

When the VCSEL operates with more than one transverse mode $(I>4.28 \mathrm{~mA})$, we study the dynamics of each transverse mode by introducing the etalon to select the mode. As the current is slightly above the threshold of the $\mathrm{LP}_{11}^{s}$ mode, a distinguishable low-frequency $(<100 \mathrm{MHz})$ peak appears in the power spectrum of the $x$ polarization of both $\mathrm{LP}_{01}$ and $\mathrm{LP}_{11}^{s}$ modes as shown in Fig. 3. Figure 3 illustrates operating modes and their dynamics for $I$ $=4.80 \mathrm{~mA}$. The polarization resolved optical spectra of the VCSEL [Fig. 3(a)] show that the $\mathrm{LP}_{01}$ and $\mathrm{LP}_{11}^{s}$ modes are essentially $x$-polarized, while the $\mathrm{LP}_{11}^{c}$ mode is $y$-polarized. Because the $\mathrm{LP}_{11}^{c}$ mode is much weaker than the other two modes and the etalon causes additional 
(a)

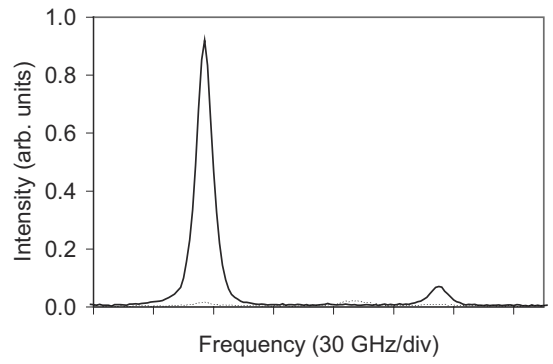

(b)

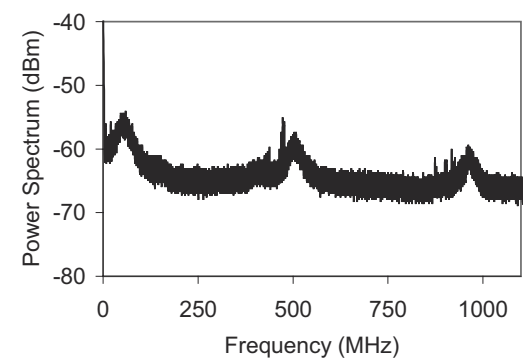

(d)

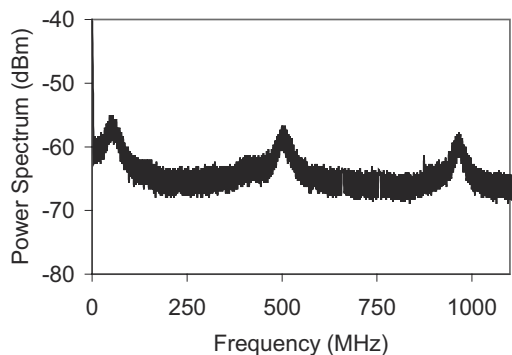

(c)

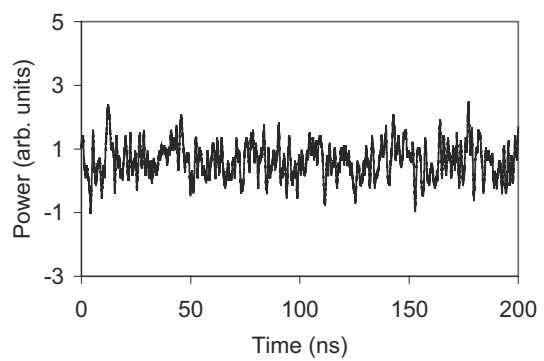

(e)

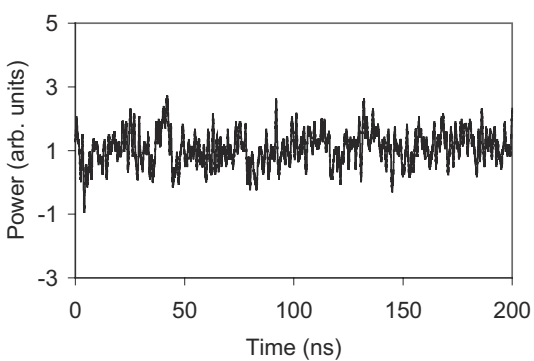

Fig. 3. Dynamics of individual transverse modes for $4.8 \mathrm{~mA}$ and the strongest feedback. (a) Polarization resolved optical spectra of the VCSEL, where the solid and dashed curves are for $x$ and $y$ polarization, respectively. (b) Power spectrum and (c) time trace of the $x$-polarization of the $\mathrm{LP}_{01}$ mode. (d) Power spectrum and (e) time trace of the $x$-polarization of the $\mathrm{LP}_{11}^{s}$ mode.

power loss, we could not measure the dynamics of the $\mathrm{LP}_{11}^{c}$ mode. Figures $3(\mathrm{~b})$ and $3(\mathrm{~d})$ are power spectra of the $x$-component of the $\mathrm{LP}_{01}$ and $\mathrm{LP}_{11}^{s}$ modes, respectively; both manifest a clear peak located at $\sim 57 \mathrm{MHz}$. In Fig. $3(\mathrm{~b})$, the peaks located at around $480 \mathrm{MHz}$, and its harmonic corresponds to external cavity resonance. However, there is a substructure at the external cavity frequency: a narrower peak at $\sim 470 \mathrm{MHz}$ and a wider peak at $\sim 504 \mathrm{MHz}$. The splitting of the peaks at the external cavity frequency has been known in single mode VCSELs and edge-emitting lasers (e.g., [25,26]), in both LFF and $\mathrm{CC}$ regimes. In some cases, the value of frequency splitting is roughly consistent with a frequency mixing interpretation; however, this interpretation does not work for our case. The frequency splitting does not appear in Fig. $3(\mathrm{~d})$, in which the frequency corresponding to the round trip in the external cavity is at $504 \mathrm{MHz}$. This may suggest that there are two external cavity modes involved in the dynamics, and the $\mathrm{LP}_{11}^{s}$ mode oscillates with the external cavity mode of higher frequency. Note that the substructure at the external cavity frequency is also observed for the $\mathrm{LP}_{11}^{s}$ mode for some other current values.

Time traces of the $x$ polarization of the $\mathrm{LP}_{01}$ and $\mathrm{LP}_{11}^{s}$ modes [Figs. 3(c) and 3(e)] manifest irregular fluctuations. Despite the clear low-frequency peak in the power spectrum of each mode, the time traces do not reveal dis- tinguishable events occurring around that frequency. This is similar to a dynamical regime (named LFF-CC regime in [15]) that represents a transition from LFF to CC regime in single-transverse-mode VCSELs and edge emitters. To evaluate the depth of fluctuation, we consider the ratio of the amplitude of the ac component to that of the dc component, which is termed normalized amplitude of the fluctuations. The normalized peak-to-peak amplitude of the fluctuations in the $x$ polarization is $\sim 3 \%$ for the $\mathrm{LP}_{01}$ mode and $\sim 50 \%$ for the $\mathrm{LP}_{11}^{s}$ mode. The greater normalized amplitude for the $\mathrm{LP}_{11}^{s}$ mode is due to the low power of the mode. Since the $\mathrm{LP}_{01}$ and $\mathrm{LP}_{11}^{s}$ modes are essentially $x$-polarized, their $x$-components represent the dynamics of the modes.

Because of the power loss in the process of mode selection, the $y$-components of the $\mathrm{LP}_{01}$ and $\mathrm{LP}_{11}^{s}$ modes are often too weak to be measured by the photodetectors. Similar difficulty appears with the $\mathrm{LP}_{11}^{c}$ mode after it is selected with the etalon. From a few examples obtained at different temperatures, we conclude that the fluctuation in the $\mathrm{LP}_{11}^{c}$ mode is much weaker. In other words, the $\mathrm{LP}_{11}^{c}$ mode seems less closely related to the dynamics of the other two transverse modes, which perhaps is attributed to their spatial profiles. From the pictures shown in Fig. 2(b), one can see that the $\mathrm{LP}_{01}$ and $\mathrm{LP}_{11}^{s}$ modes have a stronger overlap in their spatial profiles, so competition 
for spatial gain may lead to same dynamical feature in the $\mathrm{LP}_{01}$ and $\mathrm{LP}_{11}^{s}$ modes. But the $\mathrm{LP}_{11}^{c}$ mode has weaker overlap with them; more exactly, the dominant lobe of the $\mathrm{LP}_{11}^{c}$ mode is far from peaks of the other two modes. Thus the spatial competition between the $\mathrm{LP}_{11}^{c}$ mode and the other two is relatively weak, and the dynamics of the $\mathrm{LP}_{11}^{c}$ mode is less related to the dynamics of the $\mathrm{LP}_{01}$ and $L P_{11}^{s}$ modes.

As the injection current is increased, the low-frequency peak in the power spectra is stronger, and time traces demonstrate distinguishable slow events for the current ranging from 5.6 to $6.5 \mathrm{~mA}$. When the current is higher than $6.5 \mathrm{~mA}$, the slow events are not obvious, although the low-frequency peak in the power spectrum is still distinguishable. Figure 4 gives an example for $I=6.00 \mathrm{~mA}$, at which the $\mathrm{LP}_{01}$ mode is dominantly $x$-polarized and the $\mathrm{LP}_{11}^{c}$ mode is $y$-polarized; the $\mathrm{LP}_{11}^{s}$ mode has orthogonal components of comparable powers, of which the intensity of the $y$-component is about $2 / 3$ of the $x$-component. The power spectra and time traces of the $x$ component of $\mathrm{LP}_{01}$ and $\mathrm{LP}_{11}^{s}$ modes are shown in Figs. 4(b)-4(e). The rf spectra of both modes have a peak located at $\sim 81 \mathrm{MHz}$ with an amplitude significantly stronger than the peaks corresponding to external cavity resonance. The time traces show irregular fluctuations of two different time scales: a shorter time related to the round-trip time $(\sim 2.1 \mathrm{~ns})$ in the external cavity and a longer time $(\sim 12 \mathrm{~ns})$ represented by the low-frequency peak in the rf spectra. This is similar to what we reported in our previous work [18]. In particular, the slow events in the time trace of the $x$-component of the $\mathrm{LP}_{11}^{s}$ mode [Fig. 4(e)] manifest sudden power drops, and each drop is followed by gradual recovery. The normalized amplitude of the fluctuations is $\sim 30 \%$. These features are very similar to those of LFF. The fluctuations in the $x$-component of the $\mathrm{LP}_{01}$ mode display sudden power jumps followed by gradual decreases, seemingly anticorrelated with the fluctuations in the $\mathrm{LP}_{11}^{s}$ mode. This conjecture was not confirmed because the time traces in Figs. 4(c) and 4(e) were not measured simultaneously. The normalized amplitude of the fluctuation in the $\mathrm{LP}_{01}$ mode is only $5 \%$ because the mode has a high power at $6.00 \mathrm{~mA}$.

On the other hand, measurements of the $y$-component of the $\mathrm{LP}_{11}^{s}$ and $\mathrm{LP}_{01}$ modes obtained less information. The $y$-component of the $\mathrm{LP}_{01}$ mode is too weak to be detected. For the $\mathrm{LP}_{11}^{s}$ mode, a low-frequency peak at $\sim 80 \mathrm{MHz}$ is visible in the rf spectrum with an amplitude around $-61 \mathrm{dBm}$; but the peaks corresponding to external cavity resonance are minimal. The same features were observed in the rf spectrum of the $y$-polarized $\mathrm{LP}_{11}^{c}$

(a)

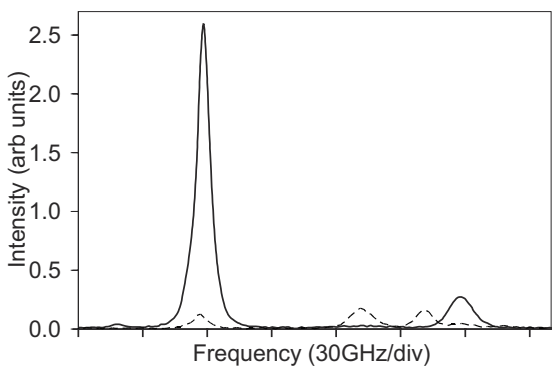

(b)

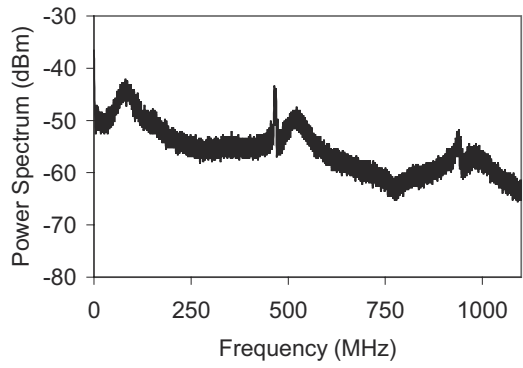

(d)

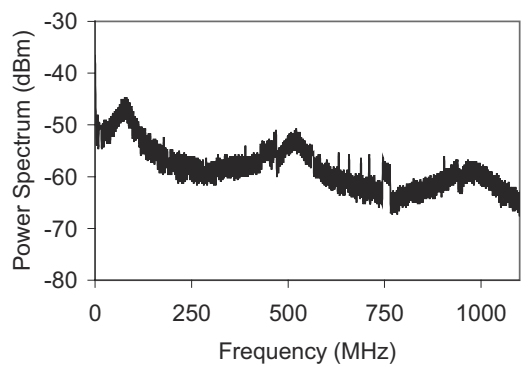

(c)

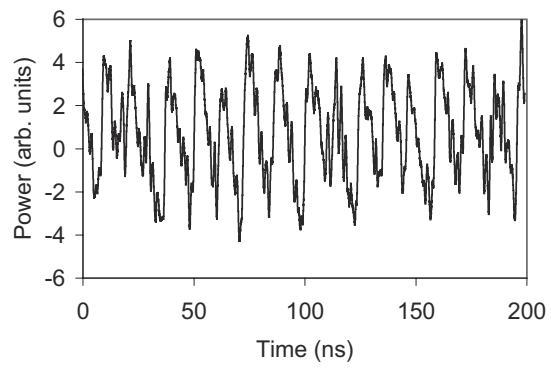

(e)

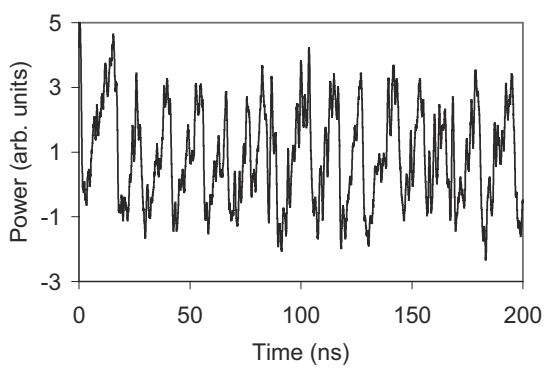

Fig. 4. Slow events in the $x$ component of $\mathrm{LP}_{01}$ and $\mathrm{LP}_{11}^{s}$ modes for $6.00 \mathrm{~mA}$ and the strongest feedback. (a) Polarization resolved optical spectral of the VCSEL, where the solid and dashed curves are for $x$ and $y$ polarization, respectively. (b) Power spectrum and (c) time trace of the $\mathrm{LP}_{01}$ mode. (d) Power spectrum and (e) time trace of the $\mathrm{LP}_{11}^{s}$ mode. The weak peaks in (d) between 600 and $800 \mathrm{MHz}$ are noises. The features of the time trace of the $\mathrm{LP}_{11}^{s}$ mode are very similar to LFF. 

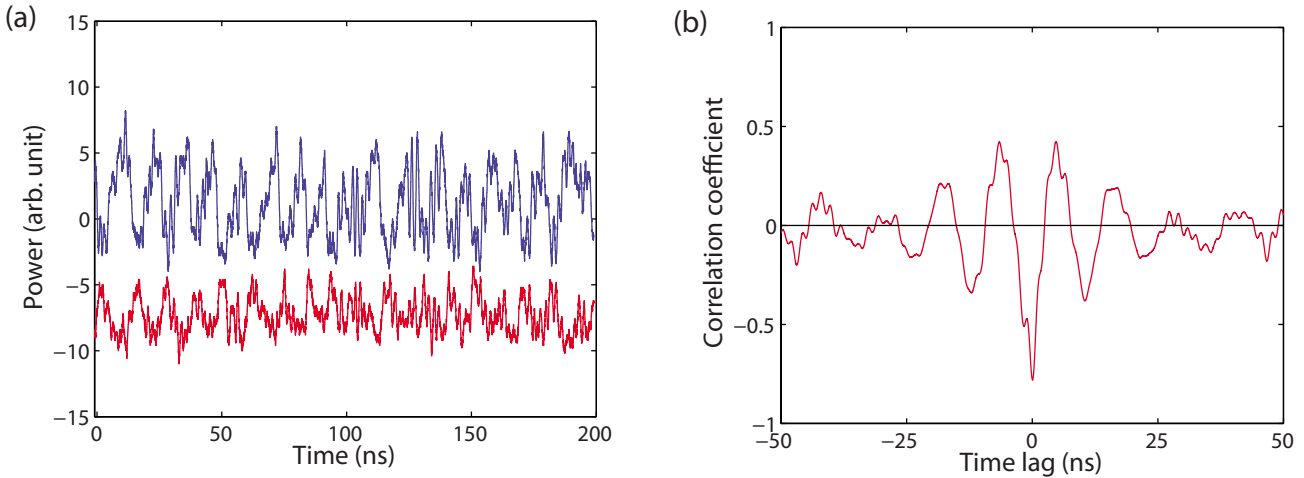

Fig. 5. (Color online) (a) Polarization resolved time traces of the total output for $6.00 \mathrm{~mA}$ and the strongest feedback, in which the upper, blue curve represents the $x$-polarized state and lower, red curve is for the $y$-polarized state. For convenience of visualization, the $y$-polarized time trace is shifted downward by 7 units. (b) The cross correlation coefficient of the orthogonally polarized states.

mode. The time traces were very near the noise level of the detectors and made it difficult to recognize any pattern in fluctuations.

The low-frequency events are also observed in the orthogonally polarized states of the total output. The $\mathrm{rf}$ spectrum of each polarization has a low-frequency peak at $81 \mathrm{MHz}$, with an amplitude of $-42 \mathrm{dBm}$ for the $x$ polarization and $-47 \mathrm{dBm}$ for the $y$ polarization. However, the amplitude of the low-frequency peak is only $-52 \mathrm{dBm}$ in the power spectrum of the total output. This suggests that the $x$ and $y$ polarizations are anticorrelated. This antiphase dynamics is confirmed by the time traces and cross correlation coefficient as shown in Fig. 5. Similar to the $x$-component of the $\mathrm{LP}_{11}^{s}$ mode, the time trace of the $x$ polarization shown in Fig. 5(a) manifests sudden power drops with gradual recovery as well as fast fluctuation related to the external cavity resonance. Again, the waveform of the slow fluctuations resembles LFF near the threshold. However, the normalized peak-to-peak amplitude of fluctuations is only $2 \%$ in the $x$ polarization and $7 \%$ in the $y$ polarization, which is much weaker than the modulation depth in LFF. The drops in the $x$ polarization are accompanied by the bursts in the $y$ polarization. The cross correlation function [Fig. 5(b)] shows a slow background modulation corresponding to the low-frequency peak in the power spectra. A fast modulation with a period of $\sim 2 \mathrm{~ns}$ is attributed to the round-trip time in the external cavity. The depth of the slow modulation is much greater than that of the fast modulation. This agrees with the rf spectra in which the low-frequency peak is significantly stronger than the peaks at the external cavity resonance and its first harmonic. The minimum value of the cross correlation coefficient is -0.78 when the time lag is zero, indicating a strong anticorrelation between the two orthogonal polarizations.

As explained above, we were unable to measure the time trace of the $y$ polarization of the $\mathrm{LP}_{01}$ and $\mathrm{LP}_{11}^{s}$ modes due to (a) the weaker power of the $y$ polarization and (b) the power loss in the process of mode selection. Although their power spectra show similarity to those of the $x$-polarized components, the correlation property of the orthogonal polarizations of each mode remains unclear. The strong anticorrelation in the orthogonally polarized states of the total output may arise from the orthogonal polarizations of each mode or from the orthogonally polar- ized transverse modes, among which $\mathrm{LP}_{01}$ and $\mathrm{LP}_{11}^{s}$ are dominantly $x$-polarized and the $\mathrm{LP}_{11}^{c}$ mode is $y$-polarized.

It is well known that the frequency and amplitude of LFF depend on control parameters $[9,12,15]$. To further compare the observed dynamics to LFF, we investigate the effects of the injection current and the strength of feedback on the low-frequency peak in the power spectrum. In the experiment, we change the effective reflectivity of the external cavity, which is proportional to the strength of feedback. The effective reflectivity of the external cavity, $R$, is the ratio of the power returning to the collimating lens to the output power. It can be changed by inserting a neutral density filter $\left(\mathrm{ND}_{3}\right.$ in Fig. 1$)$ in the external cavity. The maximum reflectivity in our setup is $25 \%$.

In Fig. 6, we map the domain of low-frequency dynamics of the $\mathrm{LP}_{01}$ and $L P_{11}^{s}$ modes in the $R$-I parameter space. For each value of $R$, we examine the lowest and highest currents for which the low-frequency peak in the

(a)

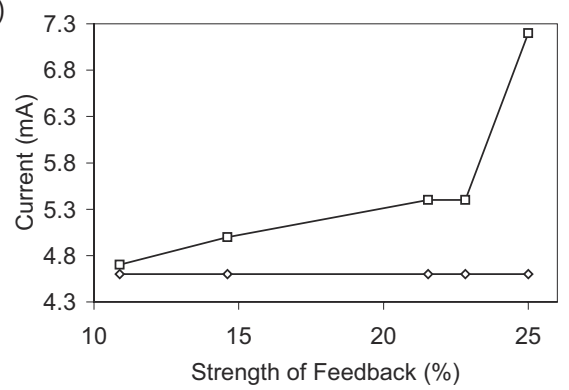

(b)

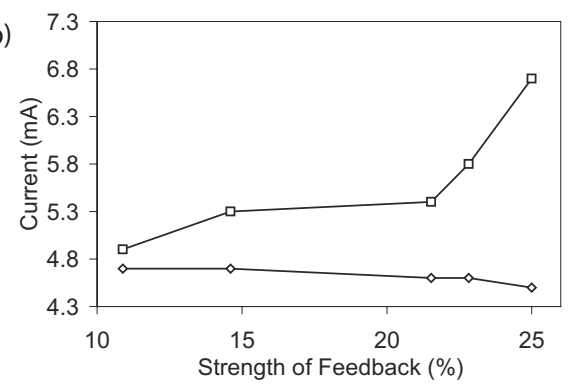

Fig. 6. Dynamical regime featured by the low-frequency peak in the power spectrum of (a) the $\mathrm{LP}_{01}$ mode and (b) $\mathrm{LP}_{11}^{s}$ mode mapped in the $R-I$ (strength of feedback-injection current) parameter space. 
power spectrum is distinguishable. The area between the two curves is where the low-frequency peak is observed. We found that the stronger the strength of optical feedback, the wider the current range for the existence of a distinguishable low-frequency peak in the power spectrum. Above the upper curve, the low-frequency peak vanishes, and a broadband feature is observed in the lowfrequency part $(<100 \mathrm{MHz})$ of the power spectrum, indicating that the VCSEL is likely in the CC regime. There is a difference between the dynamics of the $\mathrm{LP}_{01}$ and $\mathrm{LP}_{11}^{s}$ modes for the regime close but below the lower curve. For the $\mathrm{LP}_{01}$ mode, a weak shoulder near zero frequency exists in the power spectrum as well as a weak widened peak corresponding to the external cavity resonance. For the $\mathrm{LP}_{11}^{s}$ mode, there is no obvious dynamics below the lower curve. Note that the shape of the area in which the low-frequency peak is observed resembles that of the so-called LFF-CC regime shown in [15]. The similar maps are obtained at other substrate temperatures, too.

The frequency and amplitude of the low-frequency peak in the power spectrum varies with the injection current, as shown in Fig. 7. Note that the frequencies measured for the $\mathrm{LP}_{01}$ and $L \mathrm{P}_{11}^{s}$ modes are very close to each other. The frequency increases linearly with the current from 4.4 to $4.7 \mathrm{~mA}$, and then gradually approaches a constant. While the linear dependence resembles the variation of frequency of LFF versus current [9,12], the trend of approaching a constant is similar to what happened in the multi-transverse-mode regime of a VCSEL subject to polarized feedback [27]. When the current is higher than 5.0 $\mathrm{mA}$, the frequency approaches constant. This relationship is observed for several operating temperatures, ranging

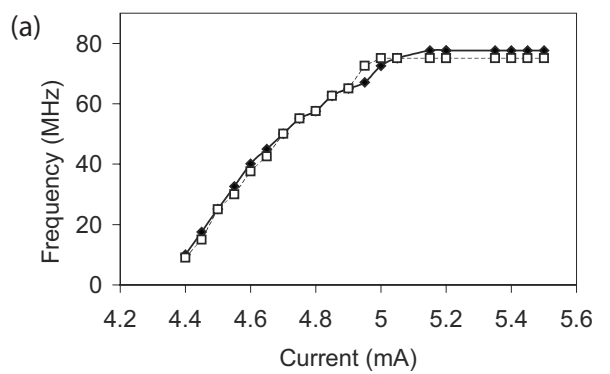

(b)

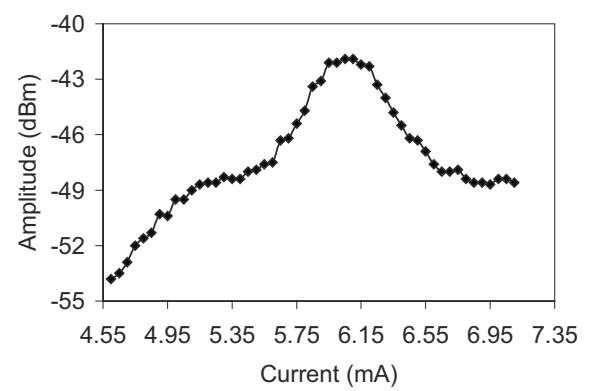

Fig. 7. (a) Frequency of the low-frequency peak in the power spectrum versus injection current, where the filled diamond represents the $L P_{01}$ mode and the blank square is for the $L P_{11}^{s}$ mode. (b) Dependence of the amplitude of the low-frequency peak in the power spectrum of the $\mathrm{LP}_{01}$ mode on the current. The peak amplitude of the $\mathrm{LP}_{11}^{s}$ mode varies with the current very similarly. For both (a) and (b), $R=25 \%$.

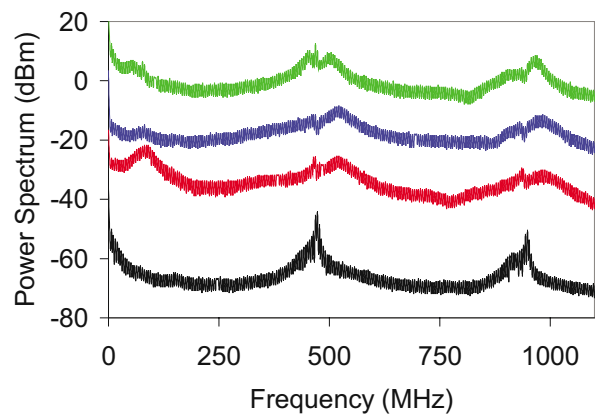

Fig. 8. (Color online) Power spectra of the $x$ polarization of the VCSEL for $I=6.00 \mathrm{~mA}$ and four substrate temperatures. The spectra from bottom to top are for $24^{\circ} \mathrm{C}$ (black), $34^{\circ} \mathrm{C}$ (red), $54^{\circ} \mathrm{C}$ (blue), and $64^{\circ} \mathrm{C}$ (green), respectively. For visual convenience, the power spectra from $34^{\circ} \mathrm{C}$ to $64^{\circ} \mathrm{C}$ are shifted up by $20 \mathrm{dBm}$ sequentially. The spectra are for $R=25 \%$.

from $24^{\circ} \mathrm{C}$ to $64^{\circ} \mathrm{C}$. The amplitude increases with increasing current, reaches a maximum value at around 6 $\mathrm{mA}$, and then decreases. This trend of variation is qualitatively similar to the result in [9]. In addition, the amplitude of the peak is significantly greater for currents ranging from $\sim 5.6$ to $6.5 \mathrm{~mA}$, which is in agreement with the current range in which distinguishable slow events are observed.

To view the influence of the substrate temperature on the dynamics, power spectra of the $x$ polarization for several temperatures and the strongest feedback are shown in Fig. 8. The injection current is chosen to be $6.00 \mathrm{~mA}$, when the VCSEL operates with three transverse modes. For $24^{\circ} \mathrm{C}$, the power spectrum has a broadband feature in the low-frequency regime $(<100 \mathrm{MHz})$ as well as peaks corresponding to the external cavity resonance. This is because the low-frequency dynamics exists in a current range less than $6 \mathrm{~mA}$ at this temperature. When $T$ $=34^{\circ} \mathrm{C}$, a peak located at $\sim 81 \mathrm{MHz}$ appears; its amplitude is greater than that of the peak at the cavity resonance. The corresponding temporal fluctuations manifest distinguishable slow events [Fig. 5(a)]. In the range of the injection current used in our experiments, this type of large-amplitude low-frequency peaks can be observed when the operating temperature ranges from $25^{\circ} \mathrm{C}$ to $36^{\circ} \mathrm{C}$ with the strongest feedback. For higher temperatures, the low-frequency peaks are still distinguishable, but their amplitudes are relatively weak as shown in the power spectra for $54^{\circ} \mathrm{C}$ and $64^{\circ} \mathrm{C}$. The time traces are similar to those shown in Fig. 3. This is probably related to the increase in the thresholds with the temperature. For a fixed injection current, the VCSEL is closer to the threshold of higher-order modes as the temperature is increased. As shown in Fig. 7(b), the amplitude of the lowfrequency peak is weaker when the VCSEL is closer to the threshold of the $\mathrm{LP}_{11}^{s}$ mode.

\section{DISCUSSION}

The experimental investigation reveals that starting slightly above the threshold of the $\mathrm{LP}_{11}^{s}$ mode, feedbackinduced dynamics demonstrate some similarity to the dynamics near the threshold of single-transverse-mode VCSELs and edge emitters. The similarity includes sev- 
eral aspects: (1) there is a distinguishable low-frequency peak in the power spectrum, although the time trace does not manifest obvious regularity; (2) when the current is increased, the low-frequency peak eventually vanishes and a broadband feature is shown in the low-frequency part of the power spectrum, while the fluctuations in the time trace look very irregular; and (3) the shape of the dynamical regime of each transverse mode in the $R-I$ parameter space resembles the LFF-CC regime shown in [15]. Therefore we conclude that there exists a LFF-CC regime above the threshold of the $\mathrm{LP}_{11}^{s}$ mode. Furthermore, for a certain current range within this regime, the amplitude of the low-frequency peak is large, and slow events become distinguishable. The time trace of the $\mathrm{LP}_{11}^{s}$ mode indicates that LFF occurs in the mode. Since fluctuations in the $x$ polarization of the total output are similar to those in the $\mathrm{LP}_{11}^{s}$ mode, it is likely that the $\mathrm{LP}_{11}^{s}$ mode causes the observed low-frequency dynamics. According to this, we infer that LFF can exist at high currents near the threshold of higher-order modes. It has been shown theoretically that LFF dynamics occurs when the system attempts to reach the maximum gain mode, which is often stable [28], and the compound cavity mode loses its stability due to a crisis with an antimode [29]. For the observed dynamics, one possible scenario is that when the $\mathrm{LP}_{11}^{s}$ mode is on, some external cavity mode is stable, making it promising for the $\mathrm{LP}_{11}^{s}$ mode to maximize its power. In that process, energy is redistributed between the $\mathrm{LP}_{11}^{s}$ mode and other modes. This can cause change in the refractive index and eventually lead to destructive interference: a drop in the intensity of the $\mathrm{LP}_{11}^{s}$ mode. Since the beam profiles of the $\mathrm{LP}_{11}^{s}$ and $\mathrm{LP}_{01}$ modes overlap, the $\mathrm{LP}_{01}$ mode fluctuates in the similar way as the $\mathrm{LP}_{11}^{s}$ mode. Whether this conjecture is correct requires a theoretical study, which is what we plan to do for the next step.

The substrate temperature influences the observed dynamics. For a fixed injection current, the dynamics changes from $\mathrm{CC}$ to $\mathrm{LFF}$ then to the LFF-CC regime when the temperature is increased from $24^{\circ} \mathrm{C}$ to $64^{\circ} \mathrm{C}$ (Fig. 8). When the temperature is less than $20^{\circ} \mathrm{C}$, the $\mathrm{LP}_{01}$ mode does not demonstrate the low-frequency dynamics. Instead, the power spectrum of the $\mathrm{LP}_{01}$ mode as well as the polarized states of the total output manifests a broadband feature in the low-frequency region, as reported in [18]. For the $\mathrm{LP}_{11}^{s}$ mode, the above dynamical regime in the $R-I$ parameter space shrinks significantly. Since the substrate temperature affects spectral gain of modes, it implies that the low-frequency dynamics may be related to the relative modal gains.

It has been shown that the frequency of the LFF increases linearly with the injection current [9,12], and the amplitude of LFF increases with the current to a maximum value and then decreases [9]. In Fig. 7, the frequency of the low-frequency peak shows a linear dependence on the current within a current range. The amplitude of the low-frequency peak reveals a similar variation with the current as the amplitude of LFF, but the maximum amplitude does not occur in the linear part of the frequency curve.

A noticeable difference between the dynamics of the
$\mathrm{LP}_{11}^{s}$ mode and the dynamical route of $\mathrm{LFF}$ is that in the latter, LFF starts very close to the threshold and evolves to CC through a LFF-CC regime as the current is increased [15]. In our observations, the LFF-CC dynamics begins from the threshold of the $\mathrm{LP}_{11}^{s}$ mode, evolves to LFF with increasing current, and then transits to the CC regime. The difference may originate from the multitransverse-mode operation. Another factor is the relatively weak strength of feedback in our experiment. As shown in [15], LFF begins near the threshold when the optical feedback is increased to a certain value. In our experiment, the strongest feedback can only reduce the threshold by $0.11 \mathrm{~mA}$ or $4 \%$; the feedback could be even weaker for the $\mathrm{LP}_{11}^{s}$ mode since its threshold is only reduced by $0.01 \mathrm{~mA}$.

Although we reported a dynamical regime similar to the LFF-CC regime before [27], there are several distinct differences between the previous work and the current one. First, different types of optical feedback-polarized feedback in [27] and isotropic feedback in this paperwere utilized, which can lead to different dynamical behaviors. Second, the experimental results in [27] were unable to tell the contribution of each transverse mode to the observed dynamics due to lack of effective ways to select modes, whereas each mode is frequency-selected and studied now. Third, the effect of the substrate temperature is investigated in this work, which yields clear evidence of LFF in the first-order mode when the injection current is high above the threshold; LFF was not observed for high currents in [27].

In conclusion, our investigation near the threshold of higher-order transverse modes reveals dynamical regimes similar to the so-called LFF-CC and LFF regimes. Fluctuations very similar to $\mathrm{LFF}$ are observed in the $\mathrm{LP}_{11}^{s}$ mode. The low-frequency dynamics is not observed when the substrate temperature is below a certain value, indicating that the modal gain plays some role in the dynamics. The similarity and difference between the observed dynamics and the dynamics near the threshold are discussed in detail. Orthogonally polarized states of the total output demonstrate similar dynamical features and are anticorrelated. The dynamics of the $\mathrm{LP}_{11}^{c}$ mode is not closely related to the other two transverse modes. This is probably attributed to weaker overlap of their spatial profiles.

\section{ACKNOWLEDGMENTS}

This work is supported by a grant to Bates College from the Howard Hughes Medical Institute. A. Valle also acknowledges Project No. TEC 2009-14581-C02-02.

\section{REFERENCES}

1. Y. C. Chung and Y. H. Lee, "Spectral characteristics of vertical-cavity surface-emitting lasers with external optical feedback," IEEE Photon. Technol. Lett. 3, 597-599 (1991).

2. S. Jiang, M. Dagenais, and R. A. Morgan, "Spectral characteristics of vertical-cavity surface-emitting lasers with strong external optical feedback," IEEE Photon. Technol. Lett. 7, 739-741 (1995).

3. P. S. Spencer, C. R. Mirasso, and K. A. Shore, "Effect of strong optical feedback on vertical-cavity surface-emitting 
lasers," IEEE Photon. Technol. Lett. 10, 191-193 (1998).

4. M. Giudici, S. Balle, T. Ackemann, S. Barland, and J. R. Tredicce, "Polarization dynamics in vertical-cavity surfaceemitting lasers with optical feedback: experiment and model," J. Opt. Soc. Am. B 16, 2114-2123 (1999).

5. H. Li, A. Hohl, A. Gavrielides, H. Hou, and K. D. Choquette, "Stable polarization self-modulation in vertical-cavity surface-emitting lasers," Appl. Phys. Lett. 72, 2355-2357 (1998).

6. K. Panajotov, M. Arizaleta, M. Camarena, H. Thienpont, H. J. Unold, J. M. Ostermann, and R. Michalzik, "Polarization switching induced by phase change in extremely short external cavity vertical-cavity surface-emitting lasers," Appl. Phys. Lett. 84, 2763-2765 (2004).

7. P. Besnard, F. Robert, M. L. Chares, and G. M. Stephan, "Theoretical modeling of vertical-cavity surface-emitting lasers with polarized optical feedback," Phys. Rev. A 56, 3191-3205 (1997).

8. C. Masoller and N. B. Abraham, "Low-frequency fluctuations in vertical-cavity surface-emitting semiconductor lasers with optical feedback," Phys. Rev. A 59, 3021-3031 (1999).

9. A. V. Naumenko, N. A. Loiko, M. Sondermann, and T. Ackemann, "Description and analysis of low-frequency fluctuations in vertical-cavity surface-emitting lasers with isotropic optical feedback by a distant reflector," Phys. Rev. A 68, 033805 (2003).

10. G. Giacomelli, F. Marin, and M. Romanelli, "Multi-timescale dynamics of a laser with polarized optical feedback," Phys. Rev. A 67, 053809 (2003).

11. N. Fujiwara, Y. Takiguchi, and J. Ohtsubo, "Observation of low-frequency fluctuations in vertical-cavity surfaceemitting lasers," Opt. Lett. 28, 896-898 (2003).

12. Y. Hong and K. A. Shore, "Influence of optical feedback time-delay on power-drops in vertical-cavity surfaceemitting lasers," IEEE J. Quantum Electron. 41, 10541057 (2005)

13. A. Torcini, S. Barland, G. Giacomelli, and F. Marin, "Lowfrequency fluctuations in vertical cavity lasers: experiments versus Lang-Kobayashi dynamics," Phys. Rev. A 74, 063801 (2006).

14. P. Besnard, B. Meziane, and G. M. Stephan, "Feedback phenomena in a semiconductor laser induced by distant reflectors," IEEE J. Quantum Electron. 29, 1271-1284 (1993).

15. T. Heil, I. Fischer, and W. Elsäßer, "Coexistence of lowfrequency fluctuations and stable emission on a single highgain mode in semiconductor lasers with external optical feedback," Phys. Rev. A 58, R2672-R2675 (1998).

16. J. Y. Law and G. P. Agrawal, "Effects of optical feedback on static and dynamic characteristics of vertical-cavity surface-emitting lasers," IEEE J. Sel. Top. Quantum Electron. 3, 353-358 (1997).
17. M. S. Torre, C. Masoller, and P. Mandel, "Transverse-mode dynamics in vertical-cavity surface-emitting lasers with optical feedback," Phys. Rev. A 66, 053817 (2002).

18. H. Lin, Z. J. Lapin, B. Malla, and A. Valle, "Polarization dynamics in a multi-transverse-mode vertical-cavity surfaceemitting laser subject to optical feedback," Phys. Rev. A 77, 033813 (2008).

19. A. Valle, H. Lin, Z. J. Lapin, and B. Malla, "Analysis of the polarization dynamics in a multitransverse-mode verticalcavity surface-emitting laser with isotropic optical feedback," Phys. Rev. A 78, 033828 (2008).

20. Y. K. Chembo, S. K. Mandre, I. Fischer, W. Elsässer, and P. Colet, "Controlling the emission properties of multimode vertical-cavity surface-emitting lasers via polarization- and frequency-selective feedback," Phys. Rev. A 79, 013817 (2009).

21. B. Tell, K. Brown-Goebeler, R. E. Leibenguth, F. M. Baez, and Y. H. Lee, "Temperature dependence of GaAs-AlGaAs vertical cavity surface emitting lasers," Appl. Phys. Lett. 60, 683-685 (1992).

22. K. D. Choquette, D. A. Richie, and R. E. Leibenguth, "Temperature dependence of gain-guided vertical-cavity surface emitting laser polarization," Appl. Phys. Lett. 64, 20622064 (1994).

23. M. Sondermann, M. Weinkath, T. Ackemann, J. Mulet, and S. Balle, "Two-frequency emission and polarization dynamics at lasing threshold in vertical-cavity surface-emitting lasers," Phys. Rev. A 68, 033822 (2003).

24. A. Valle, "Selection and modulation of higher-order transverse modes in vertical-cavity surface-emitting lasers," IEEE J. Quantum Electron. 34, 1924-1932 (1998).

25. T. Ackemann, M. Sondermann, A. Naumenko, and N. A. Loiko, "Polarization dynamics and low-frequency fluctuations in vertical-cavity surface-emitting laser subject to optical feedback," Appl. Phys. B 77, 739-746 (2003).

26. T. Heil, I. Fischer, W. Elsässer, B. Krauskopf, K. Green, and A. Gavrielides, "Delay dynamics of semiconductor lasers with short external cavities: bifurcation scenarios and mechanisms," Phys. Rev. E 67, 066214 (2003).

27. H. Lin, J. HoShue, Z. J. Lapin, and A. Valle, "Polarization instabilities in a multi-transverse-mode vertical-cavity surface-emitting laser with polarized optical feedback," Opt. Commun. 283, 1424-1433 (2010).

28. G. H. M. van Tartwijk, A. M. Levine, and D. Lenstra, "Sysyphus effect in semiconductor lasers with optical feedback," IEEE J. Sel. Top. Quantum Electron. 1, 466-472 (1995).

29. T. Sano, "Antimode dynamics and chaotic itinerancyin the coherence collapse of semiconductor lasers with optical feedback," Phys. Rev. A 50, 2719-2726 (1994). 